\title{
Use of Non-Invasive Phase Contrast Magnetic Resonance Imaging for Estimation of Atrial Septal Defect Size and Morphology: A Comparison with Transesophageal Echo \\ Chin Sze Piaw, Ong Tiong Kiam, Annuar Rapaee, Liew Chee Khoon, Liew Houng Bang, Chan Wei Ling, Hasri Samion and Sim Kui Hian
}

\section{Abstract}

Background: Transesophageal echocardiography (TEE) is a trusted method of sizing atrial septal defect (ASD) prior to percutaneous closure but is invasive, uncomfortable, and may carry a small risk of morbidity and mortality. Magnetic resonance imaging (MRI) may be useful non-invasive alternative in such patients who refuse or are unable to tolerate TEE and may provide additional information on the shape of the AOSD.

Purpose: To validate the accuracy of ASD sizing by MRI compared with TEE.

Method: Twelve patients (mean age 30 years; range 11-60 years) scheduled for ASD closure underwent TEE, cine balanced fast field echo MRI (bFFE-MRI) in four-chamber and sagittal views and phase-contrast MRI (PC-MRI) with reconstruction using the two orthogonal planes of T2-weighted images as planning. The average of the three longest measurements for all imaging modalities was calculated for each patient.

Results: Mean maximum ASD length on TEE was $18.8 \pm 4.6 \mathrm{~mm}$, mean length by bFFE-MRI was $20.0 \pm 5.0 \mathrm{~mm}$, and mean length by PC-MRI was $18.3 \pm 3.6$ $\mathrm{mm}$. The TEE measurement was significantly correlated with the bFFE-MRI and PC-MRI measurements (Pearson $r=0.69, p=0.02$ and $r=0.59, p=0.04$, respectively). The mean difference between TEE and bFFE-MRI measurements was $-1.2 \mathrm{~mm}(95 \% \mathrm{CI}:-3.7,1.3)$ and between TEE and PC-MRI was $0.5 \mathrm{~mm}$ (95\% CI: -1.9, 2.9). Bland-Altman analysis also determined general agreement between both MRI methods and TEE. The ASDs were egg-shaped in two cases, circular in 1 patient and oval in the remaining patients.

Conclusion: ASD sizing by MRI using bFFE and phase-contrast protocols correlated well with TEE estimations. PC-MRI provided additional information on ASD shapes and proximity to adjacent structures.

Keywords ASD - MRI - Phase - contrast imaging - TEE

Published in CardioVascular and Interventional Radiology, Volume 29, Number 2, 230234, 2006. 